\title{
A MODEL FOR ONE-DIMENSIONAL, NONLINEAR VISCOELASTICITY*
}

\author{
BY
}

R. C. MAC CAMY

Carnegie-Mellon University

Abstract. The problem

$$
\begin{aligned}
u_{t t} & =a(0) \sigma\left(u_{x}\right)_{x}+\int_{0}^{t} \dot{a}(t-\tau) \sigma\left(u_{x}\right)_{x} d \tau+f, \quad 0<x<1, \quad t>0, \\
u(0, t) & \equiv u(1, t) \equiv 0, \\
u(x, 0) & =u_{0}(x), \quad u_{t}(x, 0)=u_{1}(x)
\end{aligned}
$$

is considered. The essential hypotheses are that $a(t)=a_{\infty}+A(t), a_{\infty}>0, A \in L^{1}(0, \infty)$, $(-1)^{k} a^{(k)}(t) \geq 0, k=0,1,2, \sigma(0)=0, \sigma^{\prime}(\xi) \geq \epsilon>0$. It is shown that the problem has a unique classical solution for all $t$ if the data are sufficiently small and, if $f$ is suitably restricted, this solution tends to zero as $t$ tends to infinity. It is shown that the problem provides a special model for elastic materials which exhibit a memory effect.

1. Introduction. Consider the one-dimensional motion of an elastic bar. Let $x+u(x, t)$ denote the position at time $t$ of a section which is at position $x$ in the unstretched configuration. Then $u_{x}$ is a measure of strain. Nonlinear elasticity assumes that the stress $\sigma(x, t)$ at the section at time $t$ is given by $\sigma(x, t)=\sigma\left(u_{x}(x, t)\right)$. If the bar has unit length and unit density in the unstretched configuration and the ends are fixed, then an appropriate dynamic problem is:

$$
\begin{aligned}
u_{t t} & =\sigma\left(u_{x}\right)_{x}, \quad 0<x<1, \quad t>0, \\
u(0, t) & \equiv u(1, t) \equiv 0, \\
u(x, 0) & =u_{0}(x), \quad u_{t}(x, 0)=u_{1}(x) .
\end{aligned}
$$

It is known [8] that if $\sigma$ is genuinely nonlinear then this problem cannot have a global smooth solution for any non-zero data. One resolution of this paradox is to give up the requirement of smooth solutions and seek weak (or shock) solutions. This program is carried out in [5] for small data. A second idea is to change the underlying constitutive assumption. One such change is to introduce a "viscosity" term, which means assuming

$$
\sigma(x, t)=\sigma\left(u_{x}\right)+\lambda\left(u_{x}\right) u_{x t}, \quad \lambda(\xi)>0 .
$$

This alters Eq. (1.1) and it is shown in [7] and [10] that the resulting problem always has a global smooth solution which is asymptotically stable, no matter how large the data.

* Received July 24, 1976. This work was supported by the National Science Foundation. 
The model we present in this paper is intermediate between the above extremes. It arises in a natural way from the assumption of a memory effect in the material, as described below. It yields global solutions which are asymptotically stable but only if the data are sufficiently small. We conjecture that for this model data which are too large will produce breakdown.

We believe the situation is analogous to the following very simple one. Consider the equation $u_{t}+u u_{x}=0,-\infty<x<\infty, t>0$ with $u(x, 0)=\alpha\left(1+x^{2}\right)^{-1}$. This situation is analogous to (1.1)-(1.3), that is no smooth solution exists but there is a weak solution. If the equation is changed to $u_{t}+u u_{x}-\lambda u_{x x}=0, \lambda>0$, there will be a unique, global, smooth solution for any $\alpha$. If, on the other hand, the equation is replaced by $u_{t}+u u_{x}+\lambda u=0, \lambda>0$, there will be a smooth solution for $\alpha$ small but not for $\alpha$ large.

Let $L_{a}[v]$ denote the linear operator

$$
L_{a}[v](t)=\int_{0}^{t} a(t-\tau) v(\tau) d \tau .
$$

We consider the following situation:

Problem $(\mathrm{P})$. Find $u(x, t) \in C^{(2)}([0,1] \times[0, \infty))$ such that

$$
\begin{aligned}
u_{t t} & =\frac{d}{d t} L_{a}\left[\sigma\left(u_{x}\right)_{x}\right]+f, \\
u(0, t) & \equiv u(1, t) \equiv 0, \\
u(x, 0) & =u_{0}(x), \quad u_{t}(x, 0)=u_{1}(x) .
\end{aligned}
$$

We give conditions on $a, \sigma$ and $f$ which guarantee that, for sufficiently small data, (P) has a unique solution which tends to zero as $t$ tends to infinity.

Problem $(\mathrm{P})$ is very close to the one considered in [13]. The only significant difference is in the kernel $a$. In [13] we had $a \in L_{1}(0, \infty)$ while here we have $a=a_{\infty}+A, a_{\infty}>0$, $A \in L_{1}(0, \infty)$. This seemingly minor change requires a major modification of the proof in [13] and means that (P) applies to a completely different physical situation. The problem in [13] was shown to have its natural prototype in heat flow. The present problem relates to elasticity, as we indicate now.

The problem of one-dimensional motion of elastic materials with memory has been discussed at length in a series of papers by Coleman and Gurtin (see, for instance, [2]). Briefly, the situation is this. The basic assumption in the theory of fading memory for elastic materials is that the stress $\sigma(x, t)$ is given by a functional $\mathcal{F}$ of the history $u_{x}{ }^{t}$ of strain; $u_{x}{ }^{t}(\tau)=u_{x}(t-\tau), \tau \geq 0$. For each $x$ the history $u_{x}{ }^{t}$ is assumed to be in a history space $H$ defined by

$$
\begin{aligned}
H=\left\{\varphi: \varphi \text { measurable on } R^{+},|| \varphi \|_{H}\right. & =|\varphi(0)| \\
& \left.+\left(\int_{0}^{\infty} h(\tau)|\varphi(\tau)|^{p} d \tau\right)^{1 / \nu}<\infty, p \geq 1\right\},
\end{aligned}
$$

The function $h$, called the influence function, is to be non-negative and in $L_{1}(0, \infty)$. It is assumed further that $\mathcal{F}$ is continuous on $H$. An example of such a functional is

$$
\mathfrak{F}(\varphi)=\sigma(\varphi)+\int_{0}^{\infty} b(\tau) \mu(\varphi(\tau)) d \tau
$$


If $\mu$ is of algebraic growth of order $p-1$ and $b$ is suitably restricted then this functional is continuous on $H$.

We again take the density to be one and we allow an applied force $F(x, t)$ so that the equation of motion is

$$
u_{t t}=\sigma_{x}+F .
$$

We assume that the ends of the bar are fixed so that (B) holds. We assume further that the stress functional has the form (1.7). A well-set problem is to specify the history of displacement up to time $t=0$, that is $u(x, t)$ in $t \leq 0$, and have (1.S) hold for $t>0$. This yields

$$
u_{t t}=\sigma\left(u_{x}\right)_{x}+L_{b}\left[\mu\left(u_{x}\right)_{x}\right]+f \text { on } t>0 .
$$

where

$$
f(x, t)=F(x, t)+\int_{0}^{\infty} b(t+\tau) \frac{\partial}{\partial x} \mu\left(u_{x}(x,-\tau)\right) d \tau .
$$

Problem $(\mathrm{P})$ results if we specialize by assuming that

$$
\mu(\xi)=c \sigma(\xi)
$$

where $c$ is a constant. If we set $a(t)=1+c \int_{0}{ }^{t} b(\tau) d \tau$ we obtain (E) (see also [15]).

Our model thus requires that $\sigma$ and $\mu$ be essentially the same but leaves $b$ quite general. A different specialization is contained in a recent paper by Greenberg [6]. He assumes that $\sigma$ and $u_{x}$ are related by the equation

$$
\sigma_{t}+\frac{1}{T} \sigma=\left(\sigma_{1}\left(u_{x}\right)\right)_{t}+\frac{1}{T} \sigma_{2}\left(u_{x}\right) .
$$

In our context this amounts to allowing $\sigma$ and $\mu$ to be different but assuming that $b$ is a negative exponential.

If one assumes that the stress is given by a functional of the form (1.7) then relaxation experiments on materials (see [1]) indicate that one should have $\sigma(0)=\mu(0)=0$, $\sigma^{\prime}(\xi)>0, \mu^{\prime}(\xi)>0, b<0, \dot{b}>0, b \in L_{1}(0, \infty)$ and

$$
\sigma(\xi)+\mu(\xi) \int_{0}^{\infty} b(\tau) d \tau>0 .
$$

Thus for assumption (1.11) we want $\sigma(0)=0, \sigma^{\prime}(\xi)>0, c>0$ and $a(t)=a_{\infty}+A(t)$, where

$$
a_{\infty}=1+c \int_{0}^{\infty} b(\tau) d \tau>0, \quad A(t)=-c \int_{t}^{\infty} b(\tau) d \tau .
$$

We have then $a>0, \dot{a}<0$ and $\ddot{a}>0$. These are the only assumptions we use in the paper except for technical conditions on smoothness and rate of decay of $A$.

Our conditions on $a$ are the same as those used by Dafermos [4] in the study of linear viscoelasticity. Dafermos, however, studied three-dimensional situations and obtained generalized solutions. In [9] London obtains generalized solutions for some similar nonlinear problems but with very different kinds of conditions on $a$.

Sec. 2 contains a precise statement of our results and an outline of that portion of the proof which can be carried over from [13]. The proof in [13] breaks down here, where 
$a_{\infty}>0$, in the loss of a crucial energy estimate. This difficulty is remedied in Sec. 3 by establishing some new estimates. These, in turn, depend on a delicate result on linear Volterra operators which is established in Sec. 4. This last is a result of "frequency domain type" similar to those of [3], [12] and [17].

2. Statement of results and outline of proof. We first list the hypotheses on $a$. These are:

$$
\begin{gathered}
a=a_{\infty}+A(t), \quad a_{\infty}>0, \quad A \in C^{(3)}[0, \infty), \\
t^{i} A^{(k)} \in L_{1}(0, \infty), \quad j \leq 7, \quad k \leq 3, \\
(-1)^{k} A^{(k)}(t) \geq 0, \quad k=0,1,2, \quad \dot{A}(t) \not \equiv 0 .
\end{gathered}
$$

Remark 2.1. Conditions $\left(a_{3}\right)$ are standard ones in the study of Volterra differential equations $([9,14,17])$. The role of the moment conditions $\left(a_{2}\right)$ is made clear in Sec. 4 . We suspect these conditions can be weakened.

For $\sigma$ we require:

$$
\sigma \in C^{(3)}(-\infty, \infty), \quad \sigma(0)=0, \quad \sigma^{\prime}(\xi) \geq \epsilon>0 .
$$

The data $u_{0}, u_{1}$ and $f$ are subject to the following conditions:

$$
\begin{gathered}
u_{0} \in C^{(3)}[0,1], \quad u_{1} \in C^{(2)}[0,1], \\
u_{i}(0) \equiv u_{i}(1) \equiv u_{i}{ }^{\prime \prime}(0) \equiv u_{i}{ }^{\prime \prime}(1) \equiv 0, \quad i=0,1, \\
f(x, t) \in C^{(2)}([0,1] \times[0, \infty)), \\
f(0, t) \equiv f(1, t) \equiv f_{x x}(0, t) \equiv f_{x x}(1, t) .
\end{gathered}
$$

Remark 2.2. Conditions $\left(U_{2}\right)$ and $\left(F_{2}\right)$ permit smooth extensions of $u_{0}, u_{1}$ and $f$ to periodic functions on $-\infty<x<\infty$. This in turn allows one to replace $(P)$ by a pure initial-value problem (see [13]).

We also need some boundedness and growth conditions on $f$. Let us introduce some notation. We write:

$$
\begin{aligned}
|p|(T)=\sup _{0 \leq x \leq 1}|p(x, T)|, \quad|v|=\sup _{0 \leq x \leq 1}|v(x)| ; \\
((p, q))(T)=\int_{0}^{1} p(x, T) q(x, T) d x, \quad\|p\|^{2}(T)=((p, p))(T) ; \\
((v, w))=\int_{0}^{1} v(x) w(x) d x, \quad\|v\|^{2}=((v, v)) ; \\
{ }^{m}(((p, q)))^{T}=\int_{0}^{T} t^{m}((p, q))(t) d t ; \quad\left({ }^{n} \mid\|p\| \|^{T}\right)^{2}={ }^{m}(((p, p)))^{T} ; \\
(((p, q)))^{T}={ }^{0}(((p, q)))^{T} ; \quad\left\|\left|p\|\|^{T}={ }^{0}\|\mid\| p \|^{T} .\right.\right.
\end{aligned}
$$

We require that:

$$
\begin{aligned}
|f|(t) & \in L_{1}(0, \infty), \\
{ }^{i}|||f|||^{t},{ }^{i}||\left|f_{\imath}\right| \|\left.\right|^{t} & \in L_{\infty}(0, \infty), \quad j \leq 4 .
\end{aligned}
$$

All the above hypotheses are to hold throughout the paper. In addition we will assume, without loss of generality, that $a(0)=1$. 
We need the following quantities:

$$
D_{U}=\sum_{i=0}^{2} \sum_{i=0}^{1}\left|u_{i}{ }^{(j)}\right| ; \quad D_{F}=\left(|f|(t)+\left.\sum_{i=0}^{4}{ }^{i}|i| f||\right|^{t}\right)_{L_{\infty}(0, \infty)} .
$$

Theorem I. There exists a $\bar{D}$ such that if $D_{U}+D_{F}<\bar{D}$ then

(i) (P) has a unique solution $u$;

(ii) $\quad|u|(t), \quad\left|u_{t}(t)\right|, \quad\left|u_{x}(t)\right| \rightarrow 0 \quad$ as $t \rightarrow \infty$.

Remark 2.3. Theorem I can be extended to give approach to steady state. Suppose $f(x, t)=f_{0}(x)+F(x, t)$ where $F$ satisfies the conditions above. By $(\sigma)$ there exists a unique function $\bar{u}(x)$ such that

$$
\sigma\left(\bar{u}^{\prime}\right)^{\prime}=-a_{\infty}{ }^{-1} f_{0} ; \quad \bar{u}(0)=\bar{u}(1)=0 .
$$

Then one can show that $(P)$ still has a solution $u$ which has the form $u=\bar{u}+v$ where $v$ satisfies (ii) of Theorem I. The condition $a_{\infty}>0$ is crucial to the stability results. If $a_{\infty}=0$ then (i) of Theorem I is still true but (ii) is not. This can be seen by considering the linear case.

We give an outline of the proof of Theorem I. We first review the procedure of [13]. Eq. (E) can be solved for $\sigma\left(u_{x}\right)_{x}$ in the form

$$
u_{t \imath}+\frac{d}{d t} L_{k}\left[u_{\tau}\right]-\sigma\left(u_{x}\right)_{x}=f+L_{k}[f]+k(t) u_{1}(x) \equiv G(x, t)
$$

for a function $k$. This equation can be written

$$
u_{t t}+k(0) u_{t}-\sigma\left(u_{x}\right)_{x}=E,
$$

where

$$
E(x, t)=G(x, t)-\dot{k}(0) u(x, t)+\dot{k}(t) u_{0}(x)+L_{\dot{k}}[u(x, \cdot)](t) .
$$

If $E$ were known, (2.5), $(B)$ and I would be a nonlinear hyperbolic problem for $u$. The homogeneous version $(E \equiv 0)$ was considered by Nishida [16] and the inhomogeneous version in [13]. The important thing to observe is that (2.5), (2.6). (B) and (I) represents a linear perturbation of Nishida's situation. It is not difficult to establish a local existence and uniqueness theorem for this problem, but in order to obtain global results, one needs an a priori estimate. The precise result, to be established in the next section, is the following.

Theorem II. Suppose $k(0)>0$ and $\sigma$ satisfies the inequalities

$$
\left|\sigma^{(k)}(\xi)\right| \leq \bar{\sigma}, \quad k=0,1,2 \text { for all } \xi .
$$

Then there exists a $D_{0}>0, \delta>0$ and $C>0$ such that if

$$
D_{U}+D_{F}<D_{0}
$$

then any solution of $(P)$ on $[0, T]$ which satisfies

$$
\left|u_{x}\right|(T), \quad\left|u_{x t}\right|(T)<\delta
$$

also satisfies the inequalities

$$
\begin{aligned}
& T^{m}\|u\|^{2}(T), \quad T^{m}\left\|u_{x}\right\|^{2}(T), \quad T^{m}\left\|u_{t}\right\|^{2}(T), \quad T^{m}\left\|u_{x t}\right\|^{2}(T), \quad T^{m}\left\|u_{t t}\right\|^{2}(T), \\
& { }^{m}|||u|\left\|^{T}, \quad{ }^{m}||\left|u_{x}\right|\right\|^{T}, \quad{ }^{m}||\left|u_{t}\right|\left\|^{T}, \quad{ }^{m}||\left|u_{t t}\right|\right\|^{T},\left.\quad{ }^{m}||\left|u_{x t}\right|\right|^{T} \leq C, \quad m \leq 4 ;
\end{aligned}
$$




$$
\left|\frac{\partial^{i+j}}{\partial x^{i}} \frac{u}{\partial t^{j}}\right|(T) \leq C \quad i+j \leq 2 .
$$

$C$ depends only on $\left(D_{U}+D_{F}\right)$ and $C \rightarrow 0$ as $D_{U}+D_{F^{\prime}} \rightarrow 0$.

Remark 2.4. The case $m=4$ is a minimal result. If in $\left(a_{2}\right)$ we have $t^{3+N} A^{(k)} \in$ $L_{1}(0, \infty)$, then Theorem II holds for $m \leq N$. In particular, if $A(t)=0(\exp (-\beta t))$ then Theorem II holds for any $m$.

Once Theorem II is established one can complete the proof of Theorem I exactly as in [13]. First let $\sigma(\xi)$ be a function satisfying $(\sigma)$ and $\left(\sigma_{1}\right)$ and agreeing with $\sigma$ for $|\xi| \leq D_{0}{ }^{1}$. We pick $D_{0}$ in (2.7) less than $D_{0}{ }^{1}$ and solve $(P)$ for $g$. We make the data so small that (2.7) is satisfied, (2.8) is satisfied initially and $C$ in $(2.10)$ is less than $\min \left(D_{0}{ }^{1}, \delta\right)$. Then $(P)$ (with $\sigma$ ) has a local solution which continues to satisfy (2.8). Hence we can continue to arbitrary $[0, T]$. By $(2.10)$ we will have $\left|u_{x}\right| \leq D_{0}{ }^{1}$; hence $\sigma\left(u_{x}\right)=g\left(u_{x}\right)$ and we will have a solution of $(P)$.

The decay results (ii) of Theorem I are established as follows. For functions satisfying (B) we have $|u|(T) \leq\left\|u_{x}\right\|(T),\left|u_{t}\right|(T) \leq\left\|u_{x t}\right\|(T)$ and $\left|u_{x}\right|(T) \leq\left\|u_{x x}\right\|(T)$. Thus the second and fourth of the inequalities (2.9) yield the first two results of (ii). The third will be established if we can show that $\left\|u_{x x}\right\|(T) \rightarrow 0$ as $T \rightarrow \infty$. This last fact, however, follows from (2.5), (2.6), (2.9) and the following property of $k$ which is given in Lemma 3.1:

$$
k^{(i)} \in L_{1}(0, \infty) \quad j \leq 2 .
$$

The proof of Theorem I is thus reduced to that of Theorem II, and this is accomplished in the next two sections.

3. Energy estimates. In this section we give the proof of Theorem II except for a lemma to be proved in the next section. We observe first that it suffices to establish the estimates (2.9). If one has these estimates then one deduces that $\int_{0}{ }^{T}|u|(t) d t \leq C$ and hence from (2.4), (2.6) and $\left(F_{3}\right), \int_{0}{ }^{T}|E|(t) d t \leq C^{1}, C$ and $C^{1}$ independent of $T$. The estimates (2.10) then follow exactly as in [13] from arguments involving Riemann invariants.

The estimates (2.9) are established by induction on $m$, again as in [13]. The main ideas are contained in the case $m=0$ and we treat this in detail. We begin as in [13] by multiplying (2.4) by $u_{t}$ and integrating from $x=0$ to $x=1$ and $t=0$ to $t=T$. This yields, by $(\sigma)$,

$$
\begin{aligned}
\frac{1}{2}\left\|u_{t}\right\|^{2}(T)+\frac{\epsilon}{2}\left\|u_{x}\right\|^{2}(T) & \left.+\left(\left(\left(u_{t}, \frac{d}{d t} L_{k}\left[u_{\tau}\right]\right)\right)\right)\right)^{T} \\
& \leq \frac{1}{2}\left\|u_{1}\right\|^{2}+\int_{0}^{1} \int_{0}^{u_{0}^{\prime}(x)} \sigma(\xi) d \xi d x+\|\| u_{t}\|\|^{T}\|\| G \|^{T} .
\end{aligned}
$$

Lemma 3.1. The function $k$ of (2.4) satisfies:

(i) $k(0)>0$

(ii) $t^{i} k^{(n)} \in L_{1}(0, \infty) ; \quad j \leq 4, \quad n=0,1,2$,

$$
\left(\left(\left(v, \frac{d}{d t} L_{k}[v]\right)\right)\right)^{T} \geq 0 \text {. }
$$


The proof of this lemma is a minor modification of the one in [13]. The conclusion, however, is essentially different from the previous one. In [13], where $a_{\infty}=0$, we had $k=k_{\infty}+K, k_{\infty}>0$ and $K$ satisfying (ii). This had the effect of strengthening (iii) to

$$
\left(\left(\left(v, \frac{d}{d t} L_{k}[v]\right)\right)\right)^{T} \geq \alpha\left(\|\mid v\| \|^{T}\right)^{2}
$$

for some $\alpha>0$. It is clear that (3.2) and (3.1) yield a bound for $\left\|u_{t} \mid\right\|^{T}$. In the present case this estimate is lost and all the complications which follow are designed to compensate.

In the subsequent calculations we will use $C$ to denote a generic constant which is independent of $T$ and can be made small with the data and $K$ to denote absolute constants. Eqs. (3.1), (2.4), $\left(F_{4}\right)$ and Lemma 3.1 (iii) yield, then,

$$
\left\|u_{t}\right\|(T), \quad\left\|u_{x}\right\|(T) \leq C\left(1+\left.\left\|u_{t}\right\|\right|^{T}\right) .
$$

Our next step, also from [13], is to multiply (2.4) by $u$ and integrate. By $(\sigma)$, Lemma 3.1 and $\|u\|\left\|^{T} \leq \mid\right\| u_{x} \|^{T}$ this yields

$$
\frac{\epsilon}{2}\left(\|\left|u_{x}\right|||^{T}\right)^{2} \leq K\left(\|\left.\left|u_{t}\right|\right|^{T}\right)^{2}+\left(\left(u, u_{t}\right)\right)(T)+\left(\left(u_{0}, u_{1}\right)\right)+\|\| u\left\|\left.\right|^{T} i\right\| G \mid \|^{T} .
$$

In [13] where we had a bound for $\left\|u_{t} \mid\right\|^{T}$, and hence for $\left\|u_{t}\right\|(T)$ and $\left\|u_{x}\right\|(T)$, we could use (3.4) to bound $\left\|\left|u_{x}\right|\right\|^{T}$. Here we need a complicated intermediate step.

Eq. $(E)$ can be solved in a different way for $\sigma\left(u_{x}\right)_{x}$, namely,

$$
u_{t \iota}+L_{r}\left[u_{t \imath}\right]-\sigma\left(u_{x}\right)_{x}-\beta \int_{0}^{t} \sigma\left(u_{x}\right)_{x} d \tau=f+L_{r}[f],
$$

or

$$
u_{t \iota t}+\frac{d}{d t} L_{r}\left[u_{\tau \tau}\right]-\sigma\left(u_{x}\right)_{x t}-\beta \sigma\left(u_{x}\right)_{x}=f_{t}+r(0) f+L_{\dot{r}}[f] \equiv H,
$$

for any $\beta>0$ and some function $r$ depending on $\beta$. The proof of the following result is lengthy and is given in Sec. 4 .

Lemma 3.2. For any $\beta>0$ the function $r$ in (3.5) satisfies:

(i) $r(0)>0$

(ii) $\quad r=\left(\beta / a_{\infty}\right)+R, \quad t^{i} R^{(n)} \in L_{1}(0, \infty), \quad j \leq 4, \quad n=0,1,2$.

Moreover there exist positive constants $\beta, q, \alpha$ such that $q \beta<1$ and

$$
q\left(\left(\left(v, \frac{d}{d t} L_{r}[v]\right)\right)\right)^{T}-\left(\left(\left(v, L_{R}[v]\right)\right)\right)^{T} \geq(1+\alpha)\left(\|\| v \|\left.\right|^{T}\right)^{2} .
$$

Remark 3.1. Eq. (3.6) is similar to one in the paper [6] of Greenberg. It was that equation which suggested the procedure to follow.

We propose to obtain a new energy estimate by multiplying (3.6) by $q u_{t t}+u_{t}$ and integrating. Here $q$ and $\beta$ are as in Lemma 3.2. We set

$$
W[u]=u_{t t t}+\frac{d}{d t} L_{r}\left[u_{r \tau}\right] .
$$


Then one can verify the following formula:

$$
\begin{aligned}
\left(\left(\left(q u_{t \imath}+u_{t},\right.\right.\right. & W[u])))^{T}=\frac{q}{2}\left\|u_{t \imath}\right\|^{2}(T)-\frac{q}{2}\left\|u_{t \imath}(\cdot, 0)\right\|^{2} \\
& +\left(\left(u_{t}, u_{t \imath}\right)\right)(T)-\left(\left(u_{1}, u_{t \imath}(\cdot, 0)\right)\right)+\frac{\beta}{2 a_{\infty}}\left\|u_{t}\right\|^{2}(T)-\frac{\beta}{2 a_{\infty}}\left\|u_{t}\right\|^{2} \\
& +\left(\left(u_{t}, L_{R}\left[u_{\tau \tau}\right]\right)\right)(T)+q\left(\left(\left(u_{t t}, \frac{d}{d t} L_{r}\left[u_{\tau \tau}\right]\right)\right)\right)^{T} \\
& -\left(\left(\left(u_{t \iota}, L_{R}\left[u_{\tau \tau}\right]\right)\right)\right)^{T}-\left(\left\|u_{t \imath} \mid\right\|^{T}\right)^{2}
\end{aligned}
$$

We also introduce the operator

$$
Q[u]=\sigma\left(u_{x}\right)_{x t}+\beta \sigma\left(u_{x}\right)_{x},
$$

and obtain the formula

$$
\begin{aligned}
-\left(\left(\left(q u_{t t}\right.\right.\right. & \left.\left.\left.+u_{t}, Q[u]\right)\right)\right)^{T}=\frac{q}{2}\left(\left(\sigma^{\prime}\left(u_{x}\right), u_{x t}{ }^{2}\right)\right)(T)-\frac{q}{2}\left(\left(\sigma^{\prime}\left(u_{0}{ }^{\prime}\right),\left(u_{1}{ }^{\prime}\right)^{2}\right)\right) \\
& +q \beta\left(\left(\sigma\left(u_{x}\right), u_{x t}\right)\right)(T)-q \beta\left(\left(\sigma\left(u_{1}{ }^{\prime}\right), u_{1}{ }^{\prime}\right)\right)+\beta \int_{0}^{1} \int_{0}^{u_{x}\left(x, T^{\prime}\right)} \sigma(\xi) d \xi d x \\
& -\beta \int_{0}^{1} \int_{0}^{u_{0}^{\prime}(x)} \sigma(\xi) d \xi d x+\left(\left(\left(\left[(1-q \beta) \sigma^{\prime}\left(u_{x}\right)-\frac{1}{2} \sigma^{\prime \prime}\left(u_{x}\right) u_{x t}\right], u_{x t}{ }^{2}\right)\right)\right)^{r} .
\end{aligned}
$$

Since $q \beta<1$, we can choose $\delta$ so small that

$$
(1-q \beta) \epsilon-\frac{1}{2} \bar{\sigma} \delta=\tau>0,
$$

where $\bar{\sigma}$ is from $\left(\sigma_{1}\right)$. Then if $(2.8)$ is satisfied we have

$$
\left(\left(\left(\left[(1-q \beta) \sigma^{\prime}\left(u_{x}\right)-\frac{1}{2} \sigma^{\prime \prime}\left(u_{x}\right) u_{x t}\right], u_{x t}{ }^{2}\right)\right)\right)^{T} \geq \tau\left(||\left|u_{x t}\right| \|^{T}\right)^{2} .
$$

We are now ready to multiply (3.6) by $q u_{t t}+u_{t}$ and integrate. From (3.9), (3.11), $(3.13)$ and $(\sigma)$ this yields**

$$
\begin{aligned}
& \frac{q}{2}\left\|u_{t t}\right\|^{2}(T)+\left(\left(u_{t}, u_{t}\right)\right)(T)+\left(\left(u_{t}, L_{R}\left[u_{\tau \tau}\right]\right)\right)(T)+\alpha\left(\left\|\left|u_{t}\right|\right\|^{T}\right)^{2} \\
& +\frac{q \epsilon}{2}\left\|u_{x}\right\|^{2}(T)+q \beta\left(\left(\sigma\left(u_{x}\right), u_{x}\right)\right)(T)+\tau\left(\|\| u_{x}\|\|^{T}\right)^{2} \\
& \leq C+\left(q\left\|\left|u_{t t}\right|||^{T}+\right\||| u_{t} \mid \|^{T}\right)\|\mid H\| \|^{T} .
\end{aligned}
$$

We estimate the left side. By (3.3), Lemma 4.2 and $\left(\sigma_{1}\right)$ we have

$$
\begin{aligned}
\left(\left(u_{t}, u_{t}\right)\right)(T) & \leq C\left\|u_{t t}\right\|\left(T^{T}\right)\left(1+\left.\left\||| u_{t t}\right\|^{T}\right|^{T},\right. \\
\left(\left(u_{t}, L_{R}\left[u_{r \tau}\right]\right)\right)(T) & \leq\left. C\left\|u_{t t}\right\|\right|^{T}\left(1+\left\|\mid u_{t t}\right\|^{T}\right), \\
q \beta\left(\left(\sigma\left(u_{x}\right), u_{x t}\right)\right)(T) & \leq C\left\|u_{x t}\right\|(T)\left(1+\left\|\left|u_{t t} \|^{T}\right|^{T}\right) .\right.
\end{aligned}
$$

It follows that for sufficiently small data the left side of (3.14) is bounded below by

** Recall that $\mathrm{C}$ stands for any constant which is small with the data. 


$$
\frac{q}{4}\left\|u_{t t}\right\|^{2}(T)+\frac{q \epsilon}{4}\left\|u_{x t}\right\|^{2}(T)+\frac{\alpha}{2}\left(\left.\left\||| u_{t t}\right\|\right|^{T}\right)^{2}+\tau\left(\|\left|u_{x t}\right|||^{T}\right)^{2}-C .
$$

We have $\left\||| u_{t}\left|\left\|^{T} \leq\right\|\right|\left|u_{x t}\right|\right\|^{T}$. Hence, by $\left(F_{4}\right)$, we can choose the data so small that the right side of (3.14) is bounded by

$$
C+\frac{\alpha}{4}\left(\left\|\left|u_{t \imath} \|\right|^{T}\right)^{2}+\frac{\tau}{2}\left(\left\|\left|u_{x t} \|\right|^{T}\right)^{2} .\right.\right.
$$

Now (3.14) implies that (3.16) is less than or equal to (3.17). Thus we conclude that

$$
\left\|u_{t t}\right\|(T), \quad\left\|u_{x t}\right\|(T), \quad\left\|\mid u_{t}\right\|\left\|^{T}, \quad\right\| u_{x t}\|\|^{T} \leq C .
$$

The inequalities (3.3) and $\|u\|(T) \leq\left\|u_{x}\right\|(T)$, together with (3.18), yield the boundedness of $\|u\|(T),\left\|u_{t}\right\|(T)$ and $\left\|u_{x}\right\|(T)$. From (3.18) and $\left\|\left|u_{t}\|\|^{T} \leq\|\| u_{x t}\right|\right\|^{T}$ we conclude that $\left\||| u_{t} \mid\right\|^{T}$ is bounded and then, finally, (3.4) yields the boundedness of $\left\|\left|u_{x}\right|\right\|^{T}$ and hence of \|\|$u\|\|^{T}$. This concludes the verification of (2.8) for $m=0$.

The remaining portion of the induction proof is tedious but reasonably straightforward and is similar to one in [13]. The idea is to perform the same three steps as above but multiplying by $t^{m}$ as well. A typical calculation is the following:

$$
\begin{aligned}
-{ }^{m}\left(\left(\left(u_{t \iota}, \sigma\left(u_{x}\right)_{x \iota}\right)\right)\right)^{T}= & \frac{T^{m}}{2}\left(\left(\sigma^{\prime}\left(u_{x}\right), u_{x \iota}{ }^{2}\right)\right)\left(T^{\prime}\right) \\
& \quad-\frac{1}{2}\left({ }^{m}\left(\left(\left(\sigma^{\prime \prime}\left(u_{x}\right), u_{x \iota}{ }^{3}\right)\right)\right)^{T}\right)-\frac{m}{2}\left({ }^{m-1}\left(\left(\left(\sigma^{\prime}\left(u_{x}\right), u_{x \iota}{ }^{2}\right)\right)\right)^{T}\right) .
\end{aligned}
$$

If we assume that the estimates (2.9) hold for $m-1$, then the last term in (3.19) is bounded by $C$. We have also

${ }^{m}\left(\left(\left(u_{t t}, W[u]\right)\right)\right)^{T}=\frac{T^{m}}{2}\left\|u_{t \imath}\right\|^{2}(T)+{ }^{m}\left(\left(\left(u_{t t}, \frac{d}{d t} L_{r}\left[u_{r r}\right]\right)\right)\right)^{T}-\frac{m}{2}\left({ }^{m-1}\left|\left\|u_{t t}\right\|\right|^{T}\right)^{2}$.

The last term on the right is bounded by the induction hypothesis. For the second term on the right we can use the following result from [13]:

$$
{ }^{m}\left(\left(\left(u_{t t}, \frac{d}{d t} L_{r}\left[u_{\tau \tau}\right]\right)\right)\right)^{T}=\left(\left(\left(t^{m / 2} u_{t t}, \frac{d}{d t} L_{r}\left[\tau^{m / 2} u_{\tau \tau}\right]\right)\right)\right)^{r}+\Delta,
$$

where

$$
|\Delta| \leq K_{m}{ }^{m}||\left|u_{t}\right|\left\|| ^ { T } \sum _ { i = 0 } ^ { n - 1 } { } ^ { i } \left|\left\|u_{t \imath} \mid\right\|^{T},\right.\right.
$$

for some constant $K_{m}$. (This formula holds if $t^{i} \dot{r} \in L_{1}(0, \infty)$ for $j \leq m$ and this is the case here by Lemma 4.2.) The sum in (3.22) is bounded by the induction hypothesis so that $\dot{R}$ is bounded by $C\left(\left.{ }^{m}||\left|u_{t t}\right|\right|^{T}\right)$.

If one uses formulas of the type (3.19) and (3.20), together with the fact that quadratic terms dominate linear terms, one finds that the truth of (2.9) for $m-1$ implies its truth for $m$ and the proof is complete.

4. Proof of Lemma 2.2. It can be seen from (3.5) and $(E)$ that the function $r$ is to be such that the solution $\zeta$ of the equation

$$
\zeta+L_{i}[\zeta]=\varphi
$$


satisfies

$$
\zeta(t)+\beta \int_{0}^{t} \zeta(\tau) d \tau=\varphi(t)+L_{r}[\varphi](t) .
$$

If we formally take Laplace transforms of (4.1) and (4.2) we find

$$
\hat{r}(s)=\frac{1}{s a \hat{(s)}}\left(1+\frac{\beta}{s}\right)-1,
$$

where $\hat{r}$ and $\hat{a}$ are the transforms of $r$ and $a$. We study the properties of the function $r^{\wedge}$ given by (4.3).

Conditions $\left(a_{1}\right)$ and $\left(a_{2}\right)$ imply that

$$
\hat{a^{\hat{n}}}(s)=a_{\infty} s^{-1}+\hat{A}(s), \quad \hat{A} \in C^{(7)}(\operatorname{Re} s \geq 0),
$$

with $A^{-}$analytic in Re $s>0$. (One sees now the role of the moment conditions $\left(a_{2}\right)$; differentiating $a^{-}$corresponds to transforming powers of $t$ times $a$.)

It follows from Theorem 4.1 of [14] and Theorem 2.2 of [11] that conditions $\left(a_{3}\right)$ imply

$$
\begin{aligned}
\operatorname{Re} \hat{A}(i \eta)>0 & -\infty<\eta<+\infty, \\
-\eta \operatorname{Im} \hat{a}(i \eta)>0 & -\infty<\eta<+\infty .
\end{aligned}
$$

From these equations and the maximum principle for harmonic functions, it is easy to see that $s \hat{a}(s) \neq 0$ in $\operatorname{Re} s \geq 0, s \neq 0$ and hence that $\hat{r}(s)$ is defined for these $s$ values. A little closer analysis, using (4.4), shows that in fact

$$
\hat{r^{\prime}}(s)=\frac{\beta}{a_{\infty} s}+\hat{R}(s), \quad \hat{R} \in C^{(6)}(\operatorname{Re} s \geq 0) .
$$

Conditions $\left(a_{1}\right)$ and $\left(a_{2}\right)$ and integration by parts yield the estimates

$$
\hat{a^{\hat{n}}}(s)=a(0) s^{-1}+\dot{a}(0) s^{-2}+\ddot{a}(0) s^{-3}+o\left(s^{-3}\right),
$$

as $s \rightarrow \infty$ in $\operatorname{Re} s \geq 0$. From (4.3) we obtain, then,

$$
\hat{r}(s)=r_{0}(\beta) s^{-1}+r_{1}(\beta) s^{-2}+o\left(s^{-2}\right) \text { as } s \rightarrow \infty,
$$

where

$$
r_{0}(\beta)=\beta-\dot{a}(0)>0, \quad r_{1}(\beta)=\dot{a}(0)^{2}-\ddot{a}(0)-\beta \dot{a}(0) .
$$

One now constructs $r$ by taking the inverse transform, that is,

$$
r(t)=\frac{\beta}{a_{\infty}}+\frac{1}{2 \pi i} \int_{\gamma-i \infty}^{\gamma+i \infty} e^{s t} \hat{R}(s) d s \equiv \frac{\beta}{a_{\infty}}+R(t) .
$$

The properties of $\hat{\gamma}$ can be used to show that the integral is independent of $\gamma$ in $\gamma \geq 0$ and defines a continuous function $R$ of $t$ with $r(0)=r_{0}(\beta)$. Moreover, one can show that $r$ satisfies (4.2). The behavior of $R$ for large $t$ can be determined by taking $\gamma=0$ in (4.11) and using the Riemann-Lebesgue lemma. This estimate can be improved by integrating by parts in (4.11). Conditions $\left(a_{1}\right)$ and $\left(a_{2}\right)$ can be used to show that the estimates (4.9) can be differentiated and then six integrations by parts in (4.11) and the Riemann-Lebesque lemma yield the estimate

$$
R(t)=O\left(t^{-6}\right) \text { as } t \rightarrow \infty .
$$


Finally we observe that the transforms of $\dot{R}$ and $\ddot{R}$ are $s R^{\wedge}-R(0)$ and $s^{2} R^{\wedge}-s R(0)-$ $\dot{R}(0)$ respectively and the whole analysis can be applied to these as well to show that $\dot{R}$ and $\ddot{R}$ also satisfy (4.12).

The analysis above follows closely procedures in [12] and [13] and yields immediately conclusions (i) and (ii) of Lemma 3.2. Conclusion (iii) is harder and we turn to it now.

Eq. (3.7) is equivalent to the inequality

$$
M[v: T] \equiv q \int_{0}^{T} v(t) \frac{d}{d t} L_{r}[v](t) d t-\int_{0}^{T} v(t) L_{R}[v](t) d t \geq(1+\alpha) \int_{0}^{T} v^{2}(t) d t .
$$

We verify (4.13) for $v$ 's such that $v \in C^{(2)}[0, T], v(0)=0$. Since these are dense in $L_{2}[0, T]$, (4.13) will then be true for all $v \in L_{2}[0, T]$. For the special $v$ s we set $v_{T}(t)=v(t), 0 \leq$ $t \leq T, v_{T}(t)=0$ for $t<0$ or $t>T$. Then $v_{T}$ has a Fourier transform $v_{T}^{\sim} \in L_{2}(-\infty, \infty)$. Formula (2.9) of [11] and Lemma 4.3 of [14] then yield the representation

$$
M[v: T]=\frac{1}{\pi} \int_{0}^{\infty}(-q \eta \operatorname{Im} \hat{r}(i \eta)-\operatorname{Re} \hat{R}(i \eta))\left|v_{T}^{\sim}(\eta)\right|^{2} d \eta .
$$

We note that $\operatorname{Re} R^{\wedge}(i \eta)=\operatorname{Re} \hat{r}^{\wedge}(i \eta)$. Thus it follows from (4.14) and Parseval's theorem that (3.7) will be implied by the following result.

Proposition 4.1. There exist positive constants $q, \beta, \alpha$ such that $q \beta<1$ and

$$
-q \eta \operatorname{Im} \hat{r}(i \eta)-\operatorname{Re} \hat{r}(i \eta) \geq 1+\alpha .
$$

Proof: We have, by (4.3),

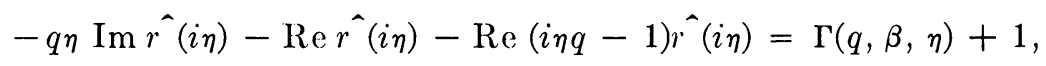

where

$$
\Gamma(q, \beta, \eta)=\left(a_{r}^{\hat{}}(i \eta)^{2}+{a_{i}}^{\hat{(}}(i \eta)^{2}\right)^{-1}\left\{\left(q+\frac{\beta}{\eta^{2}}\right) a_{r}^{\hat{r}}(i \eta)+\frac{1-q \beta}{\eta} a_{i} \hat{(}(i \eta)\right\} .
$$

Here we have written $\hat{a}(i \eta)=a_{r}(i \eta)+i a_{i}(i \eta)$. Eq. (4.15) then requires $\Gamma(q, \beta, \eta) \geq \alpha$ for all $\eta$.

We write (4.17) in the form

$$
\Gamma(q, \beta, \eta)=\varphi(\eta) \gamma(q, \beta, \eta),
$$

where

$$
\begin{gathered}
\varphi(\eta)=-\frac{a_{i}^{\hat{(}}(i \eta)}{\eta}\left(\hat{a_{r}} \hat{(i \eta)^{2}}+\hat{\left.a_{i} \hat{(}(i \eta)^{2}\right)^{-1}},\right. \\
\gamma(q, \beta, \eta)=q \beta-\frac{q \eta a_{r} \hat{(}(i \eta)}{a_{i} \hat{(}(i \eta)}-\frac{\beta a_{r} \hat{(i \eta)}}{\eta a_{\hat{i}} \hat{(}(i \eta)}-1 .
\end{gathered}
$$

We show first that there exists $\alpha_{1}>0$ such that

$$
\varphi(\eta) \geq \alpha_{1} \text { for all } \eta \text {. }
$$

Eq. (4.6) shows that $\varphi(\eta)>0$ for all $\eta$ and since $\varphi$ is a continuous function, it suffices to establish (4.21) for large $\eta$. But it follows immediately from (4.8) that $\varphi(\eta) \rightarrow(1 / a(0))$ as $\eta \rightarrow \infty$. Hence (4.21) holds.

We have thus reduced Proposition (4.1) to showing that $\gamma$ is bounded below by a 
positive constant. We write

$$
m(\eta)=\frac{\eta a_{r} \hat{(i \eta)}}{a_{i}{ }^{\hat{}}(i \eta)} ; \quad n(\eta)=\frac{1}{\eta^{2}} m(\eta),
$$

so that $\gamma(q, \beta, \eta)=q \beta-m(\eta) q-n(\eta) \beta-1$. From (4.4) and (4.8) we have

$$
\begin{aligned}
& m(\eta) \rightarrow 0, \quad n(\eta) \rightarrow-\frac{\hat{A} \hat{(}(0)}{a_{\infty}} \text { as } \quad \eta \rightarrow 0, \\
& m(\eta) \rightarrow \dot{a}(0), \quad n(\eta) \rightarrow 0 \quad \text { as } \quad \eta \rightarrow \infty .
\end{aligned}
$$

We consider the curves $\gamma(q, \beta, \eta)=0$ in the $q-\beta$ plane, $q>0$. These can be written

$$
\beta=\beta_{\gamma}(q: \eta)=\frac{1+m(\eta) q}{q-n(\eta)} .
$$

We have

$$
\frac{1}{q}-\beta_{\gamma}=\frac{-m(\eta)-n(\eta) q^{2}}{q(q-n(\eta))}
$$

From (4.22), (4.5) and (4.6) we see that $m(\eta)<0$ and $n(\eta)<0$ for all $\eta$; hence $(1 / q)-$ $\beta_{\gamma}>0$ for all $\eta$. We establish a stronger result. Given $\bar{\epsilon}$ and $\bar{q}, \bar{Q}, 0<\bar{q}<\bar{Q}<\infty$, we claim that there exists an $\bar{\epsilon}(\bar{q}, \bar{Q})>0$ such that,

$$
\frac{1}{q}-\beta_{\gamma} \geq \bar{\epsilon} \text { for all } q, \bar{q} \leq q \leq \bar{Q} \text { and all } \eta \text {. }
$$

If this were not true then we could find sequences $q_{k} \rightarrow q, \bar{q} \leq q \leq \bar{Q}, \eta_{k} \rightarrow \infty$ with $\left(1 / q_{k}\right)-\beta_{\gamma}\left(q_{k}, \eta_{k}\right) \rightarrow 0$. But from (4.23), (4.24) and (4.26), (1/ $\left.q_{k}\right)-\beta_{\gamma}\left(q_{k}, \eta_{k}\right) \rightarrow$ $-\dot{a}(0) / q^{2}>0$, a contradiction.

We now proceed as follows. Choose $0<\bar{q}<\bar{Q}<\infty$ and take $\epsilon<\bar{\epsilon}(\bar{q}, \bar{Q})$. Then choose $q$ and $\beta$ such that

$$
\bar{q} \leq q \leq \bar{Q}, \quad q \beta<1, \quad(1 / q)-\beta<\epsilon / 2 .
$$

By (4.27) we have, then,

$$
\beta>\frac{1}{q}-\frac{\epsilon}{2} \geq \beta_{\gamma}+\bar{\epsilon}-\frac{\epsilon}{2}>\beta_{\gamma}(q, \eta) \text { for any } \eta .
$$

But by (4.20) we have $\partial \gamma / \partial \beta=q-n(\eta)>0$ and thus (4.29) implies

$$
\gamma(q, \beta, \eta)>\gamma\left(q, \beta_{\gamma} \eta\right)=0 \text { for any } \eta \text {. }
$$

Eq. (4.30) shows that $\gamma(q, \beta, \eta)$ is positive for all $\eta$. Once again it is a continuous function of $\eta$ so if we can show that it has a positive limit as $\eta \rightarrow \infty$ it will necessarily have a positive lower bound. But from (4.20) and (4.22) we have $\gamma(q, \beta, \eta) \rightarrow q \beta+$ $\dot{a}(0) q>0$ as $\eta \rightarrow \infty$. This concludes the proof of Proposition 4.1 and hence of Lemma 3.2.

\section{REFERENCES}

[1] D. R. Bland, The theory of linear viscoelasticity, Pergamon Press, New York, 1960

[2] B. D. Coleman and M. E. Gurtin, Waves in materials with memory. II. On the growth and decay of one-dimensional acceleration waves, Arch. Rat. Mech. 19, 239-265 (1965) 
[3] C. Corduneanu, Integral equations and stability of feedback systems, Academic Press, New York, 1973

[4] C. M. Dafermos, An abstract Volterra equation with applications to linear viscoelasticity, J. Diff. Eq. 7, 554-569 (1970)

[5] James Glimm and P. D. Lax, Decay of solutions of systems of nonlinear hyperbolic conservation laws, Mem. Amer. Math. Soc. 101 (1970)

[6] J. M. Greenberg, A-priori estimates for flows in dissipative materials (preprint)

[7] J. M. Greenberg, R. C. MacCamy and V. J. Mizel, On the existence, uniqueness and stability of solutions of the equation $\rho-\chi_{t t}=E\left(\chi_{x}\right) \chi_{x x}+\lambda \chi_{x x}$, J. Math. Mech. 17, 707-728 (1968)

[8] P. D. Lax, Development of singularities of solutions of non-linear hyperbolic differential equations, J. Math. Phys. 5, 611-613 (1964)

[9] Stig-Olaf London, An existence result on a Volterra equation in a Banach space (preprint)

[10] R. C. MacCamy, Existence uniqueness and stability of $u_{t t}=\partial / \partial x\left(\sigma\left(u_{x}\right)+\lambda\left(u_{x}\right) u_{x}\right)$, Indiana Univ. Math. J. 20, 231-338 (1970)

[11] R. C. MacCamy, Nonlinear Volterra equations on a Hilbert space, J. Diff. Eq. 16, 373-393 (1974)

[12] R. C. MacCamy, Remarks on frequency domain methods for Volterra integral equations, J. Math. Anal. Appl.

[13] R. C. MacCamy, An integro-differential equation with applications in heat flow, Q. Appl. Math. (this issue)

[14] R. C. MacCamy and J. S. W. Wong, Stability theorems for some functional equations, Trans. Amer. Math. Soc. 164, 1-37 (1972)

[15] R. R. Nachlinger and L. T. Wheeler, Wave propagation and uniqueness in one-dimensional simple materials, J. Math. Anal. Appl. 48, 294-300 (1974)

[16] T. Nashida, Global smooth solutions for the second order quasilinear equation with first-order dissipation (preprint)

[17] J. A. Nohel and D. F. Shea, Frequency domain methods for Volterra equations (preprint) 